\title{
Novel adipokine asprosin modulates browning and adipogenesis in white adipose tissue
}

\author{
Yanli Miao ${ }^{1, *}$, Haojie Qin ${ }^{1, *}$, Yi Zhong1, Kai Huang1 and Caijun Rao 2 \\ 1Department of Cardiology, Union Hospital, Tongji Medical College, Huazhong University of Science and Technology, Wuhan, China \\ 2Department of Geriatrics, Tongji Hospital, Tongji Medical College, Huazhong University of Science and Technology, Wuhan, China
}

Correspondence should be addressed to K Huang or C Rao: unionhuang@yeah.net or caijunrao@sina.com

*(Y Miao and H Qin contributed equally to this work)

\begin{abstract}
Obesity is an increasingly serious epidemic worldwide characterized by an increase in the number and size of adipocytes. Adipose tissue maintains the balance between lipid storage and energy utilization. Therefore, adipose metabolism is of great significance for the prevention, treatment and intervention of obesity. Asprosin, a novel adipokine, is a circulating hormone mainly secreted by white adipose tissue. Previous studies have shown that asprosin plays a role in fasting-induced homeostasis, insulin resistance, and glucose tolerance. However, whether it can regulate the metabolism of adipose tissue itself has not been studied. This study intended to examine the roles and potential mechanisms of asprosin in adipose regulation. We first demonstrated that the expression level of asprosin was significantly downregulated in subcutaneous white adipose tissue (scWAT) of high-fat diet (HFD)-fed or cold-stimulated mice. Overexpression of asprosin in ScWAT reduced heat production, decreased expression of the browning marker uncoupling protein 1 (UCP1) and other browning-related genes, along with upregulation of adipogenic gene expression. Mechanistically, we found that Nrf2 was activated upon cold exposure, but this activation was suppressed after asprosin overexpression. In primary cultured adipocytes, adenovirusmediated asprosin overexpression inhibited adipose browning and aggravated lipid deposition, while Nrf2 agonist oltipraz could reverse these changes. Our findings suggest that novel adipokine asprosin negatively regulated browning and elevate lipid deposition in adipose tissue via a Nrf2-mediated mechanism. Asprosin may be a promising target for the prevention and treatment of obesity and other metabolic diseases.
\end{abstract}

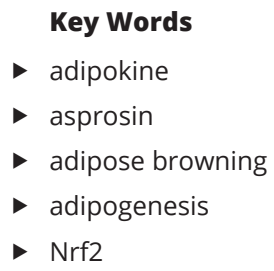

Journal of Endocrinology (2021) 249, 83-93

\section{Introduction}

Obesity and obesity-related disorders such as type 2 diabetes are steadily increasing worldwide with the improvement of people's living standards and unhealthy lifestyle (Mohammed et al. 2018). Obesity is a pathophysiological condition that can lead to a variety of diseases, such as type 2 diabetes (Bhupathiraju \& $\mathrm{Hu}$ 2016), cardiovascular diseases (Vecchie et al. 2018), non-alcoholic fatty liver disease (Polyzos et al. 2019) and even increased risk of cancers (Salaun et al. 2017). If energy intake exceeds energy expenditure, the energy imbalance will eventually lead to obesity (McQueen et al. 2018).

Tackling obesity requires reducing calorie intake first, followed by changes in metabolic efficiency (Camilleri \& Acosta 2018), such as reducing fat synthesis and 
increasing energy expenditure in key metabolic organs, such as adipose tissue. Adipose tissue can be further divided into white (WAT) and brown (BAT) adipose tissue, which are distinct in form and function and have different metabolic characteristics (Bartelt \& Heeren 2014). When exposed to certain environmental stimuli (such as cold exposure), adipocytes in white adipose tissue convert to a brownish phenotype, which is called white adipose tissue browning (Wang \& Seale 2016). The browned white adipocytes begin to express uncoupling protein-1 (UCP1), a specific marker of brown adipose tissue, making white adipose tissue more likely to generate heat and increase the body's energy consumption. In addition to the change of environmental temperature, many factors such as inflammation, oxidative stress and autophagy can affect the white adipose browning (Huerta-Delgado et al. 2020, Yamamuro et al. 2020). Browning of white adipose tissue is currently considered as a new approach to weight loss, insulin sensitivity and glucose tolerance, with potential therapeutic effects in the treatment of obesity and cardiovascular diseases.

It is well known that white adipose tissue is the main endocrine organ that secretes a variety of 'adipokines' including leptin, adiponectin, resistin, and asprosin (Fasshauer \& Bluher 2015). Asprosin, the c-terminal propeptide of FBN1 (Muthu \& Reinhardt 2020), is a novel adipokine secreted mainly by white adipose tissue (Romere et al. 2016) and plays an important regulatory role in the glucose metabolism of liver (Greenhill 2016), muscle (Jung et al. 2019) and pancreas (Lee et al. 2019, Wang \& $\mathrm{Hu}$ 2020). Asprosin is also a centrally acting orexigenic hormone that increases appetite and ultimately leads to obesity and weight gain (Duerrschmid et al. 2017). However, its metabolic regulation on adipose tissue itself has not been studied.

Our findings indicated that the expression of asprosin was decreased during the browning process of white adipose tissue, while asprosin overexpression in white adipose tissue inhibited its browning, reduced the body's thermogenesis, increased fat synthesis, and aggravated the lipid deposition in adipocytes by inhibiting the Nrf2 pathway. This physiological function of asprosin may make it a new therapeutic target for obesity and related diseases.

\section{Materials and methods}

\section{Animals}

All animal work was approved by the Institutional Animal Care and Use Committee of Huazhong University of Science and Technology. Fifty 10-week-old male C57BL/6J mice were randomly divided into two groups and administered adenoviruses $\left(1 \times 10^{9}\right.$ plaque forming units) (Longchamp et al. 2018) expressing the entire coding sequence of murine asprosin (Ad-Asprosin) or a negative control virus encoding green fluorescent protein (Ad-GFP) in the groin subcutaneous white adipose tissue. Adenoviruses were injected into the center of inguinal scWAT pads within a marked area of approximately $0.5 \mathrm{~cm}$ in diameter. The injection site on each side was divided into 10 intensive sites to ensure that the adipose tissue in the labeled area could be overexpressed. After adenovirus injection, the mice were housed under $12 \mathrm{~h}$ light:12 $\mathrm{h}$ darkness cycle with ad libitum access to food and drinking water in a controlled temperature $\left(23^{\circ} \mathrm{C} \pm 1^{\circ} \mathrm{C}\right)$ for 2 days. Two days later, we exposed the mice to cold exposure $\left(4^{\circ} \mathrm{C}\right)$. In the first $6 \mathrm{~h}$, we measured their rectal temperature using a mouse thermometer at specified time points. After $24 \mathrm{~h}$ of acclimation (Huang et al. 2017), indicators related to respiratory metabolism were measured using Comprehensive Lab Animal Monitoring System (CLAMS) for the next $24 \mathrm{~h}$. At day 5, the mice were sacrificed and tissues were collected to further examine the expression of asprosin and histomorphology. Adenovirus was recommended a period of 3-7 days following infection to assess effects on gene expression or physiology (GomezBanoy \& Lo 2017).

HFD mice were fed with a diet containing 60\% calories as fat (D12492, Research Diets, New Brunswick, $\mathrm{NJ}$ ) for 12 weeks. Other mice used in this study were fed with the standard chow diet (D12450B, Research Diets, New Brunswick, NJ).

\section{Generation of recombinant adenovirus}

Replication-defective recombinant adenovirus carrying the entire coding sequence of asprosin (Ad-Asprosin) with a signal peptide was constructed with the Adenovirus Expression Vector Kit (Takara Bio Inc., Kusatsu, Japan). Asprosin is a novel adipokine encoded by exon 65 and exon 66 of Fibrillin 1 (FBN1). In order to ensure its normal function and secretion, we constructed adenovirus encoding signal peptide (1-24aa) and propeptide (25-44aa), which can be found on UniProt (Q61554). An adenovirusonly-containing green fluorescence protein (GFP) was used as a negative control (Ad-GFP). Amplification and purification of recombinant adenovirus was designed and synthesized by OBiO Technology (Shanghai, China) Corp., Ltd. 


\section{Metabolic analysis}

Mice were individually kept in metabolic cages at $4^{\circ} \mathrm{C}$ under a $12 \mathrm{~h}$ light: $12 \mathrm{~h}$ darkness cycle, starting at 08:00 h. After $24 \mathrm{~h}$ of acclimation, the oxygen consumption rate $\left(\mathrm{VO}_{2}\right)$, carbon dioxide production rate $\left(\mathrm{VCO}_{2}\right)$, and physical activity were measured using Comprehensive Lab Animal Monitoring System (CLAMS) for the next $24 \mathrm{~h}$, and each cage was monitored at a 15 min interval.

\section{Enzyme-linked immunosorbent assay}

Enzyme-linked immunosorbent assay (ELISA) was performed to measure asprosin levels in mice serum by using commercial kits (Bioswamp, MU30851) according to the manufacturer's instruction. Read the absorbance at $450 \mathrm{~nm}$ immediately after adding the stop solution. Quantitative results were calculated by standard curves.

\section{Western blot analysis}

Cells or tissues were homogenized in ice-cold RIPA buffer with a proteinase inhibitor cocktail (Sigma-Aldrich). Protein concentrations were determined using the BCA Protein assay kit (Thermo Scientific). Equal amounts of protein were fractionated by $10 \%$ SDS polyacrylamide gels, followed by immunoblotting with the following primary antibodies: asprosin (FNab09797), UCP1 (ab10983; Abcam), PGC1a (ab54481; Abcam), $\beta$-tubulin (ab6046; Abcam). Membranes were then incubated with peroxidase-conjugated secondary antibody, and specific bands were detected with a Bio-Rad (Hercules, CA) imaging system.

\section{RNA extraction and qRT-PCR}

Total RNA was extracted from cells or tissues with the use of TRIzol reagent (D9108A, Takara Bio). RNA was reverse-transcribed using the RNA PCR Kit (RR036A, Takara Bio). Quantitative PCR (qPCR) amplification was performed with an ABI PRISM 7900 Sequence Detector system (Applied Biosystem, Foster City, CA) according to the manufacturer's instructions. Relative gene expression (normalized to 18S) was calculated using the comparative CT method formula $2^{-\Delta \Delta C T}$. The real-time PCR primer sequences are shown in Supplementary Table 1 (see section on supplementary materials given at the end of this article).

\section{Histological analysis}

Histology experiments were performed exactly as described previously (Rao et al. 2019). Hematoxylin and eosin staining (H\&E) of subcutaneous white adipose tissue samples were fixed in $4 \%$ paraformaldehyde overnight and embedded in paraffin. Paraffin blocks were sliced into $5 \mathrm{~mm}$ in thickness and stained with hematoxylin and eosin for $5 \mathrm{~min}$. For IHC experiments, paraffin slides were incubated with $0.3 \% \mathrm{H} 2 \mathrm{O} 2$ for $10 \mathrm{~min}$ to quench the endogenous peroxidase, then incubated in 1\% BSA (BSA) for $10 \mathrm{~min}$, followed by incubation with UCP1-antibody at $4 \mathrm{C}$ overnight. Afterwards, the slides were incubated with horseradish peroxidase (HRP)-conjugated secondary antibody at room temperature for $2 \mathrm{~h}$. The images were photographed on a light microscope with imagepro-plus program.

\section{MEF culture, differentiation, and treatment}

Primary Mouse embryonic fibroblasts (MEFs) were generated from pregnant mice at day 13.5 post coitum as described before (Braga et al. 2013). The embryonic head and internal organs were removed, the remaining carcasses were rinsed in $1 \times$ PBS and minced with scissors. Next, minced carcasses were suspended with $20 \mathrm{ml}$ $0.025 \%$ trypsin/EDTA (Roche) in a $50 \mathrm{ml} \mathrm{EP}$ tube, and incubated in a water bath for an hour at $37^{\circ} \mathrm{C}$. The tube was shaken every $15 \mathrm{~min}$. The trypsin was neutralized by adding DMEM with $10 \%$ FBS, then passed through a $100 \mu \mathrm{m}$ nylon mesh cell strainer into a new tube to remove undigested tissues. Cell suspensions were centrifuged and resuspended in Dulbecco's modified Eagle medium (DMEM) supplemented with 10\% fetal bovine serum (FBS) and 1\% penicillin and streptomycin. The cells were plated into a $10 \mathrm{~cm}$ dish and cultured until confluent. Two days after confluence (day 0), the medium was changed to preadipocyte differentiation medium (DMEM supplemented with 10\% FBS, 3-isobutyl-1methylxanthine (0.5 mM, I5879, Sigma-Aldrich), insulin $(10 \mu \mathrm{g} / \mathrm{ml}, 1342106$, Sigma-Aldrich), dexamethasone $(2 \mu \mathrm{M}$, D1756, Sigma-Aldrich), and rosiglitazone (2.5 $\mu \mathrm{M}$, R2408, Sigma-Aldrich). From day 2, medium containing $10 \mu \mathrm{g} / \mathrm{mL}$ insulin and $2.5 \mu \mathrm{M}$ rosiglitazone was changed every 2 days. The cells were divided into two groups and were infected with adenovirus on day 6 and the culture medium was changed to $2 \%(\mathrm{w} / \mathrm{v})$ fatty acidfree BSA on day 8. Cells were treated with or without 8-Br-cAMP (1 mM, B5386, Sigma) for 4 and $12 \mathrm{~h}$ after $24 \mathrm{~h}$ pre-incubation with Oltipraz (10 $\mu \mathrm{M}, \mathrm{HY}-12519, \mathrm{MCE})$.

This work is licensed under a Creative Commons Attribution 4.0 International License. 
All cells were maintained in a $37^{\circ} \mathrm{C}$ and $5 \% \mathrm{CO} 2$ humidified atmosphere.

\section{Red Oil O staining}

Cell slides were stained with Oil Red O (O0625, Sigma) and counterstained with Mayer's hematoxylin to visualize intracellular lipid droplets. All digital images were obtained with a light microscope (Olympus, Tokyo, Japan).

\section{Statistical analysis}

Values are expressed as means of triplicate experiments \pm S.E.M. Each series of experiments was repeated at least three times. Exclusion of individual data points was determined using an outlier calculator included in the Prism 7 software package (GraphPad Software Inc., CA) and excluded from analyses. Statistical analysis was performed using a Student's t-test when two groups were compared or by one-way ANOVA, followed by Bonferroni post hoc tests, if multiple groups were compared. $P$ value $<0.05$ was considered statistically significant.
A
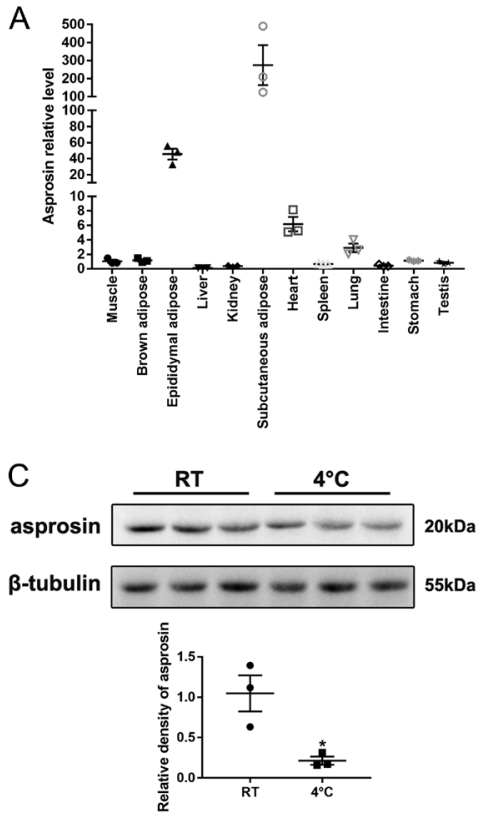

B

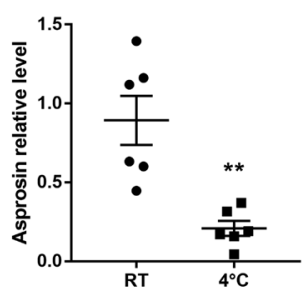

D

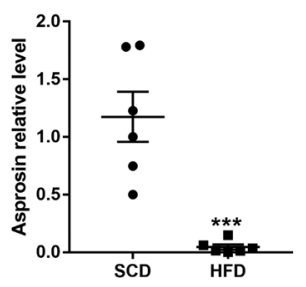

\section{Figure 1}

Decreased expression of asprosin in mouse white adipose tissue (WAT). (A) mRNA expression levels and distribution of asprosin in C57/BL6] mice $(n=3)$. The mRNA (B) and protein (C) expression of asprosin in the subcutaneous white adipose tissue (scWAT) of mice at room temperature (RT) or upon cold exposure $\left(4^{\circ} \mathrm{C}\right)$ for $24 \mathrm{~h}(n=3-6)$.

(D) Asprosin mRNA level in scWAT of mice with standard chow diet (SCD) or high-fat diet (HFD) $(n=6)$. ${ }^{*} P<0.05, * * P<0.01$ and $* * * P<0.001$ compared to the control group.

\section{Results}

Asprosin is mainly expressed in white adipose tissue and decreases in adipose metabolism

Asprosin was expressed predominantly in white adipose tissue (subcutaneous adipose and epididymal adipose), with a minor fraction in brown adipose tissue (BAT), liver, and muscle (Fig. 1A). To further explore whether asprosin was involved in adipose browning, we examined its expression levels in different models (cold exposure and high-fat diet, classic models of browning). First, we analyzed asprosin expression in subcutaneous white adipose tissue (scWAT) from mice maintained at room temperature (RT) or in a cold environment $\left(4^{\circ} \mathrm{C}\right)$ and found that the expression of asprosin was decreased, at both mRNA and protein levels (Fig. 1B and C). Furthermore, as revealed in Fig. 1D, in the subcutaneous tissue of mice fed with high-fat diet (HFD) for 12 weeks, the expression of asprosin was significantly decreased, compared to that of standard chow diet (SCD) control mice. These findings indicated that the novel adipokine asprosin may play an important role in adipose energy production and conversion.

\section{Asprosin overexpression decreases energy expenditure of mice during cold exposure}

We next determined whether asprosin overexpression affected energy balance. Recombinant adenovirus (Ad-Asprosin) were generated and locally injected into the groin subcutaneous white adipose tissue to elevate the level of asprosin in vivo (Supplementary Fig. 1B, C and D). The whole-body oxygen consumption rate $\left(\mathrm{VO}_{2}\right)$ (Fig. $2 \mathrm{~A}$ and $\mathrm{B}$ ) and carbon dioxide production rate $\left(\mathrm{VCO}_{2}\right)$ (Fig. 2C and D) decreased in Ad-Asprosin mice compared to those in Ad-GFP control mice when mice were exposed to cold. Whole-body energy expenditure, which was represented by heat production, was significantly blunted in Ad-Asprosin mice (Fig. 3E and F). The basal respiration exchange ratio (RER), indicating the relative proportion of carbohydrates vs lipids as a substrate for energy consumption (18), by calculating $\mathrm{VCO}_{2} / \mathrm{VO}_{2}$, were increased in Ad-Asprosin mice (Fig. 2G), meaning a lower availability of fatty acids. Additionally, by measuring the rectal temperature of mice, we detected that asprosin overexpression in mice adipose tissue made them more difficult in maintaining their body temperature. During cold exposure, the core temperature of Ad-GFP control mice displayed better body temperature

This work is licensed under a Creative Commons Attribution 4.0 International License. 
conservation $\left(37.73 \pm 0.28^{\circ} \mathrm{C}\right.$ to $\left.36.4 \pm 0.74^{\circ} \mathrm{C}\right)$, while the Ad-asprosin mice dropped rapidly from $37.45 \pm 0.38^{\circ} \mathrm{C}$ to $35.55 \pm 1.05^{\circ} \mathrm{C}$ in the first $6 \mathrm{~h}$ (Fig. $2 \mathrm{H}$ ), suggesting that overexpression of asprosin cut down energy expenditure via reduced thermogenesis.

\section{Asprosin overexpression inhibits the development of browning in white adipose tissue}

When stimulated by cold, white adipocytes convert to beige adipocytes. The main feature of beige adipocyte is that they produce heat, keeping the mice's body temperature stable during cold stimuli.

In order to further explore the relationship between the difficulty in maintaining body temperature and the overexpression of asprosin in mice, we analyzed the gene expression of classical browning markers including UCP1, PGC1a, PRDM16, Dio $_{2}$ and PPARa, and the results revealed that most of the markers were significantly decreased in the scWAT of the asprosin overexpression group (Fig. 3A). Meanwhile, asprosin overexpression in WAT led to an decreased adipocytes size in scWAT (Fig. 3B). IHC analysis of UCP1 staining (brown stain) of scWAT sections also showed less UCP1+ cells in Ad-Asprosin mice (Fig. 3C). Consistent with the above results, Western blot analysis of UCP1 and PGC1a, typical markers of thermogenesis, showed decreased protein levels in scWAT from Ad-Asprosin mice compared to those in Ad-GFP control mice after exposure to cold (Fig. 3D). These data demonstrated that asprosin significantly suppressed being in WAT.

\section{Nrf2 participates in asprosin mediated inhibition of adipose browning}

To uncover the underlying mechanism of the aforementioned observations, we explored whether Nrf2, a crucial antioxidant transcription factor, was involved

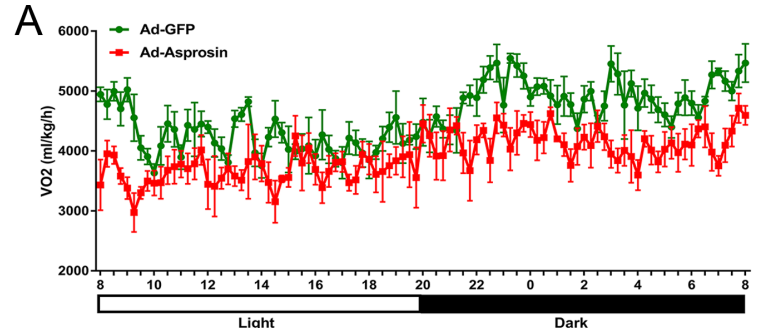

C

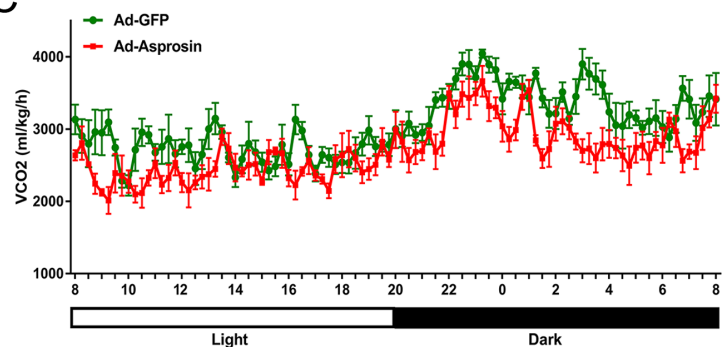

E

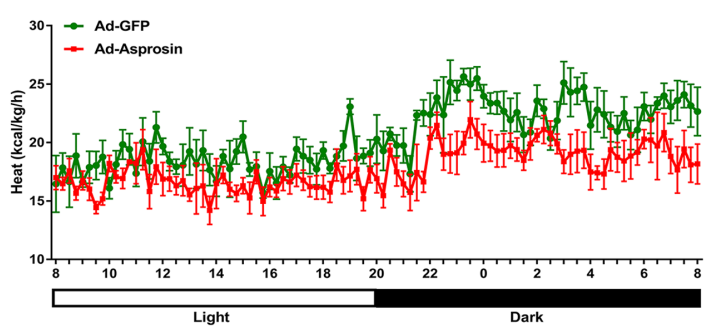

B

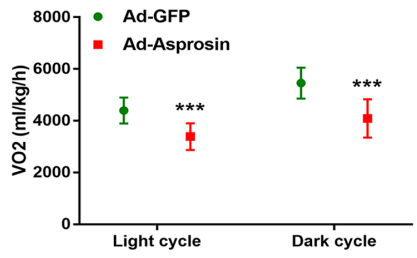

D

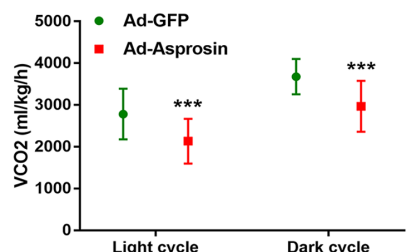

F

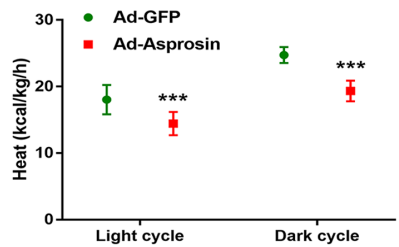

G

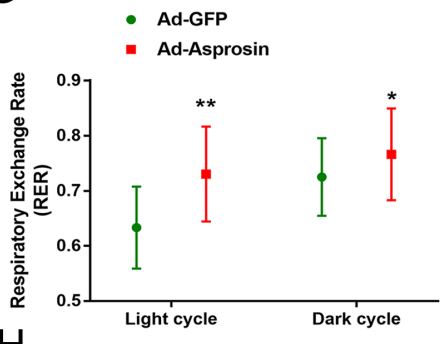

$\mathrm{H}$

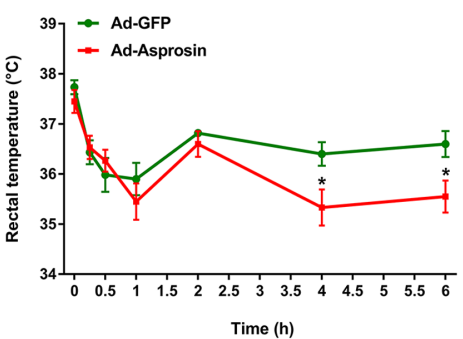

Figure 2

Asprosin overexpression decreases energy expenditure of mice during cold exposure. Mice injected with adenovirus overexpressing GFP (Ad-GFP) or asprosin (Ad-Asprosin) were housed individually at $4^{\circ} \mathrm{C}$ for a $12 \mathrm{~h}$ light: $12 \mathrm{~h}$ darkness cycle. The whole-body oxygen consumption rate $\left(\mathrm{VO} \mathrm{O}_{2}\right.$ ( $\mathrm{mL} / \mathrm{kg} / \mathrm{h}$ ) $(\mathrm{A}$ and $\mathrm{B})$, carbon dioxide production $\left(\mathrm{VCO}_{2}\right)(\mathrm{mL} / \mathrm{kg} / \mathrm{h})(\mathrm{C}$ and $\mathrm{D})$ and heat production (E and F) of mice measured by Columbus Oxymax metabolic chambers at $4^{\circ} \mathrm{C}$ and the average values were calculated by $12 \mathrm{~h}$ light: $12 \mathrm{~h}$ darkness periods. (G) The average values of respiratory exchange ratio (RER) in the mice for a $24 \mathrm{~h}$ cycle were calculated from the metabolic cage data. $(\mathrm{H})$ Rectal temperature of the mice was recorded at the indicated time points upon cold exposure $\left(4^{\circ} \mathrm{C}\right)(n=6-8) . * P<0.05, * \star P<0.01$ and $* \star \star P<0.001$ compared to the control group. A full color version of this figure is available at https://doi.org/10.1530/JOE-20-0503.

https://joe.bioscientifica.com https://doi.org/10.1530/JOE-20-0503
(C) 2021 The authors Published by Bioscientifica Ltd. Printed in Great Britain

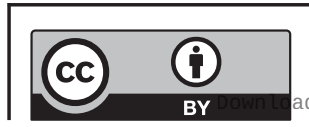

This work is licensed under a Creative Commons Attribution 4.0 International License. 
UCP1
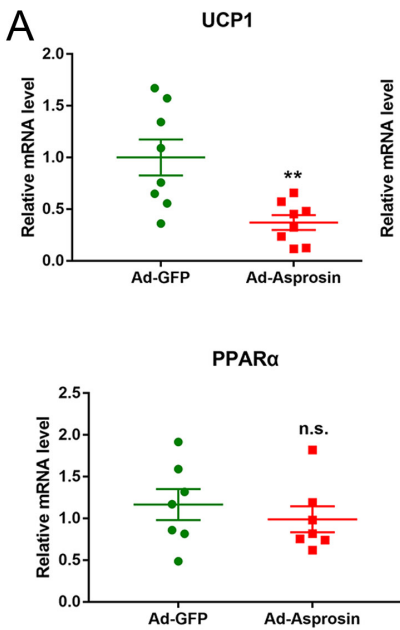

GC1 $\alpha$

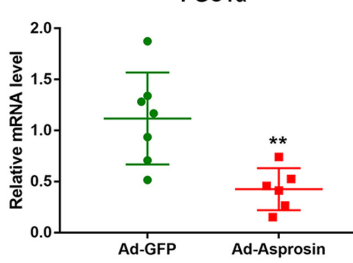

B

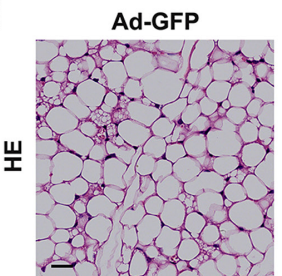

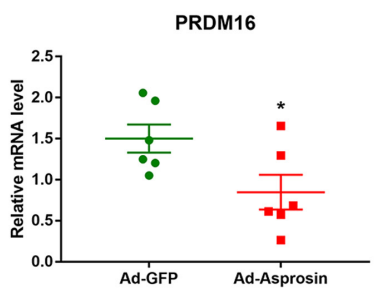
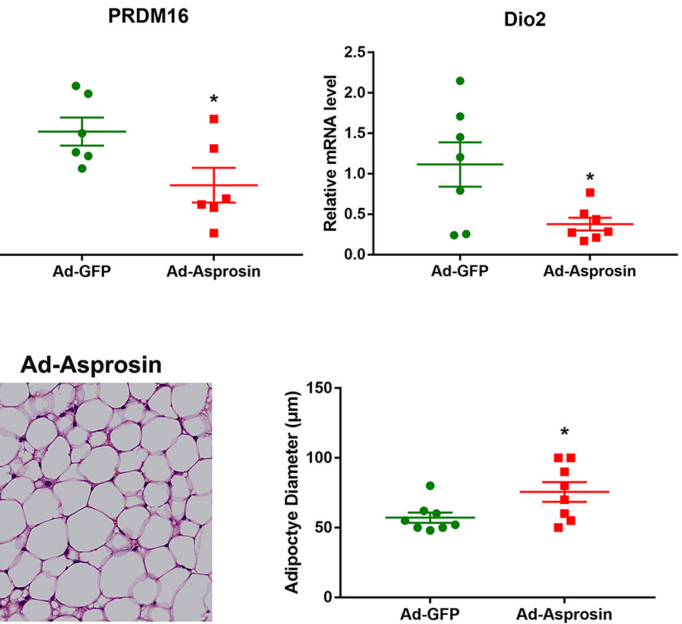

C
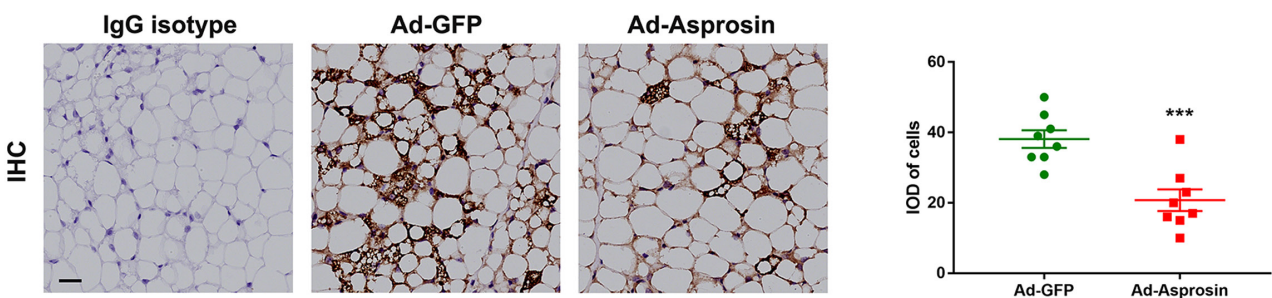

D

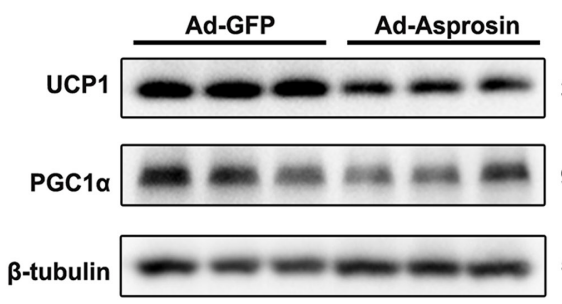

$33 \mathrm{kDa}$
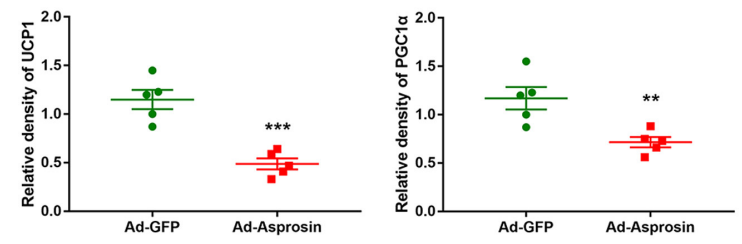

\section{Figure 3}

Asprosin overexpression inhibits the development of browning in white adipose tissue. Mice were treated as indicated in Fig. 2. (A) Effects of asprosin overexpression on the expression of adipose browning factors (UCP1, PGC1a, PRDM16, Dio ${ }_{2}$ and PPARa) in the scWAT of cold-treated mice, as determined by qRT-PCR $(n=6-8)$. (B) H\&E staining of scWAT of Ad-GFP or Ad-Asprosin mice and adipocyte size distribution. Quantification of adipocyte size was performed by ImageJ software $(n=8)$. (C) Immunohistochemistry analysis of UCP1 staining (brown stain) in scWAT sections $(n=8)$. (D) Western blot analysis for UCP1 and PGC1a in the scWAT of experimental mice $(n=5)$. ${ }^{2}<0.05, * \star P<0.01$ and $* \star \star P<0.001$ compared to the control group. n.s. means no statistical significance. A full color version of this figure is available at https://doi.org/10.1530/JOE-20-0503.

in the process. Nrf2 is a transcription factor known for its antioxidant properties, with the development of research, it has been found that Nrf2 plays a pivotal role in adipose biology, both directly and indirectly (Schneider $\&$ Chan 2013). The activation of Nrf2 is coordinated by specific repressor Kelch-like ECH-associated protein 1 (Keap1) (Wang et al. 2020). We measured the expression levels of Nrf2 and Keap1, both the mRNA and protein levels of Nrf2 were downregulated in Ad-Asprosin mice, together with its downstream target genes, however, the change of Keap1 expression level was not significant (Fig. 4A and B). To further verify the involvement of Nrf2 signaling in the cellular-autonomous regulation of asprosin on thermogenesis, cAMP and oltipraz (an Nrf2 activator) (Zhao et al. 2018) was applied to the subsequent cell experiments, cAMP is a ubiquitous second messenger that plays an important role in a wide range of cells and multiple signaling pathways. The cAMP signaling pathway is very important for early brown adipocytes differentiation by promoting the proliferation and expression of transcription factors, including key thermogenic regulators (Reverte-Salisa et al. 2019). As shown in Fig. 4C, Western blot showed that the protein levels of UCP1 were lowered by Ad-Asprosin when compared to that of the Ad-GFP control, while oltipraz could rescue the inhibition of asprosin overexpression on 
thermogenesis in adipocytes. In parallel, we also tested the effects of oltipraz on the expression of browning markers including UCP1, PGC1a, PRDM16, Dio 2 and PPARa and got a consistent conclusion (Fig. 4D). These observations suggested that asprosin refrain adipose browning through an Nrf2-mediated mechanism.

\section{Asprosin promotes adipogenesis in primary adipocytes in vitro through Nrf2 pathway}

Nrf2 also plays an important role in adipogenesis (Haider \& Larose 2020). In previous studies, we observed that overexpression of asprosin could reduce the expression of
Nrf2, inhibit its activity and downregulate its downstream target genes. Therefore, we further examined the mRNA levels of adipogenesis gene expression, including ACC1, FASN, SCD1 and SREBP, as displayed in Fig. 5A, almost all these genes were elevated in Ad-Asprosin mice when upon cold exposure except for SREBP. Consistent with the increased mRNA levels, the protein expression of ACC1 and FASN were also upregulated by Ad-Asprosin (Fig. 5B), along with higher free fatty acid (FFA) release in mice serum (Supplementary Fig. 1F). Red Oil O staining of adipocyte also showed that overexpression of asprosin increases lipid synthesis and reduces lipolysis in adipocytes, which can be reversed by Nrf2 agonists oltipraz (Fig. 5C).
A
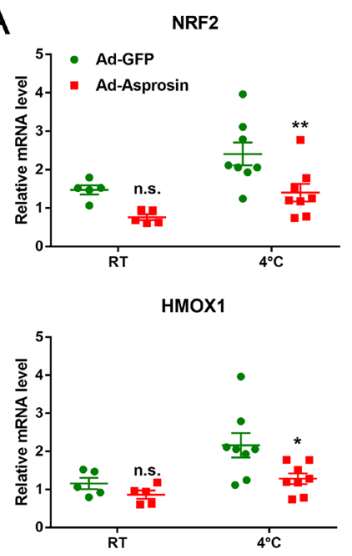
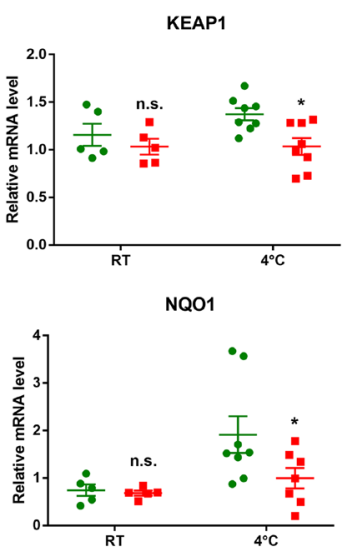

B
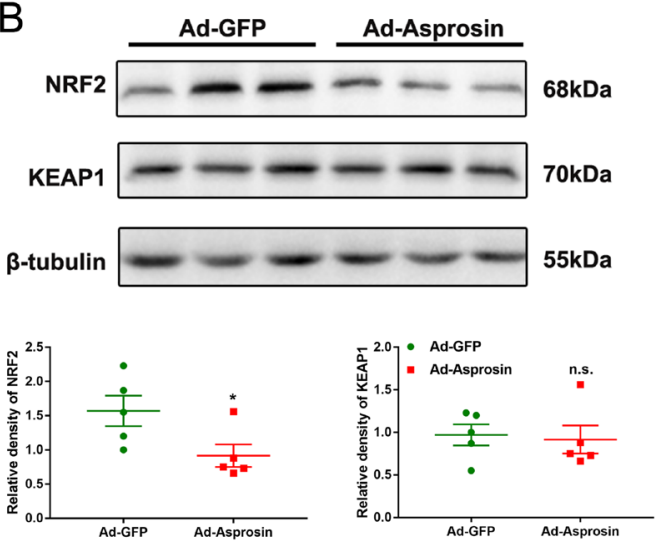

C
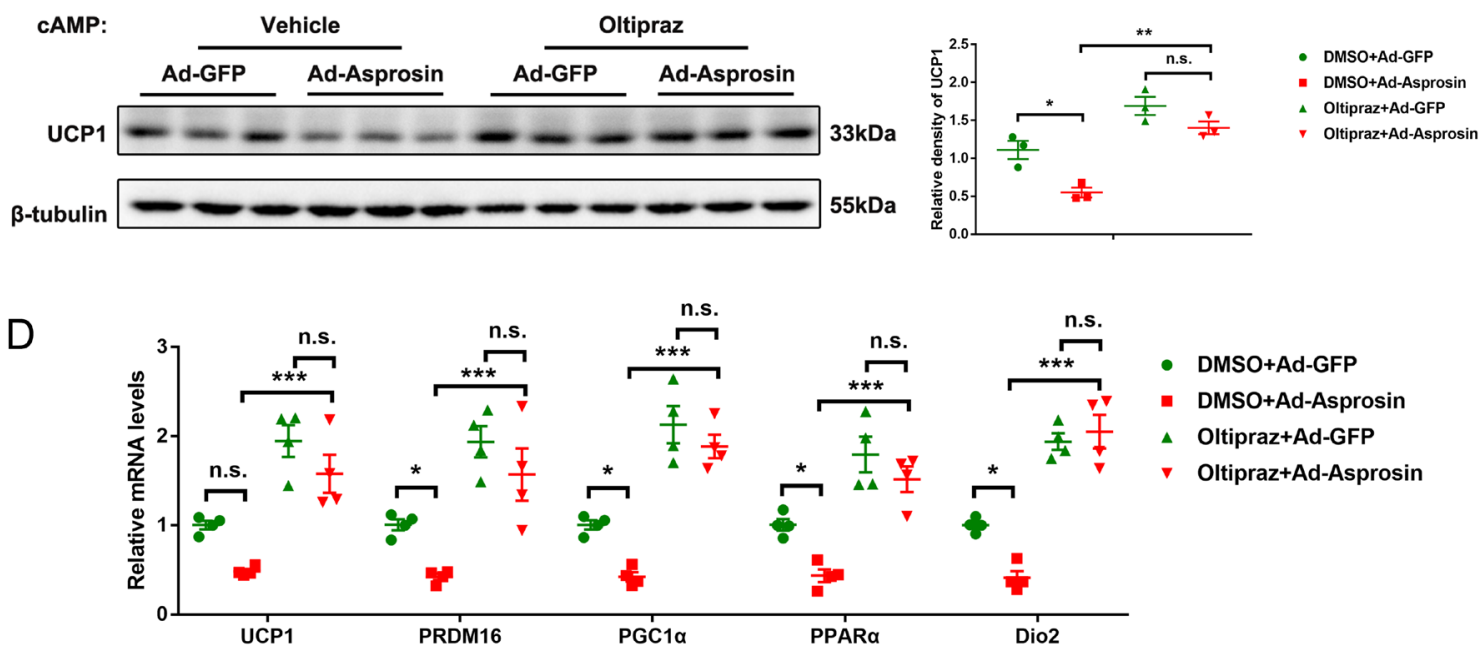

\section{Figure 4}

Nrf2 participates in asprosin mediated inhibition of adipose browning. (A) Relative mRNA levels of the Nrf2, Keap1 and Nrf2 targets Hmox1 and Nqo1 in mice scWAT. (B) Western blot analysis of Nrf2 and Keap1 in scWAT from $4^{\circ} \mathrm{C}$ treated mice $(n=5-8)$. (C and D) After adenovirus transfection, adipocytes were preincubated with oltipraz $10 \mu \mathrm{M}$ for $24 \mathrm{~h}$ and then stimulated with 8-Br-cAMP $1 \mathrm{mM}$ for $4 \mathrm{~h}$. Oltipraz treatment elevated Nrf2 activity and rescued the inhibition of asprosin overexpression on thermogenesis in response to 8-Br-cAMP in adipocytes $(n=3-4)$. (C and D) The temperature condition was RT. (C) Protein levels of UCP1 in adipocytes were detected by Western blot. (D) Relative mRNA levels of browning related genes in cultured adipocytes with different treatments. ${ }^{*} P<0.05, * \star P<0.01$ and $* \star \star P<0.001$ compared to the control group. n.s. means no statistical significance. A full color version of this figure is available at https://doi.org/10.1530/JOE-20-0503.

https://joe.bioscientifica.com https://doi.org/10.1530/JOE-20-0503
(C) 2021 The authors Published by Bioscientifica Ltd. Printed in Great Britain

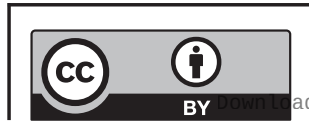

This work is licensed under a Creative Commons Attribution 4.0 International License. 

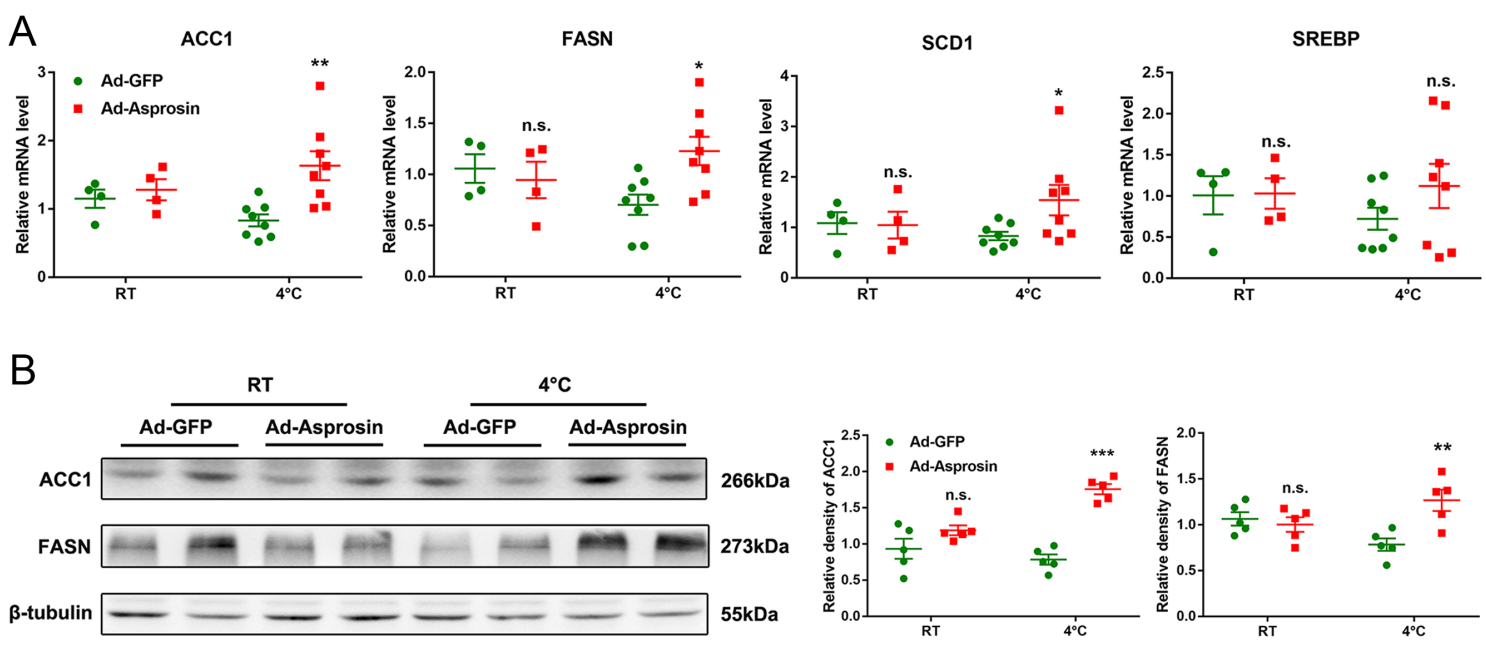

C
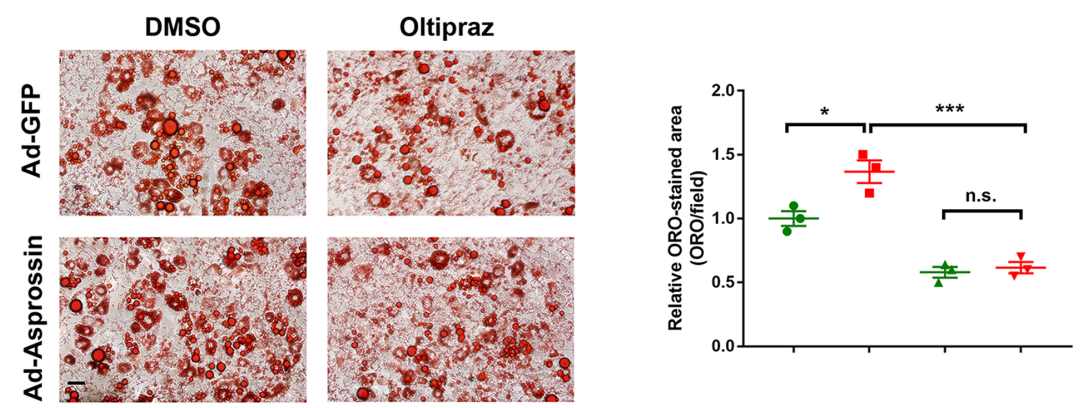

- DMSO+Ad-GFP

- DMSO+Ad-Asprosin

- Oltipraz+Ad-GFP

$\checkmark$ Oltipraz+Ad-Asprosin

D
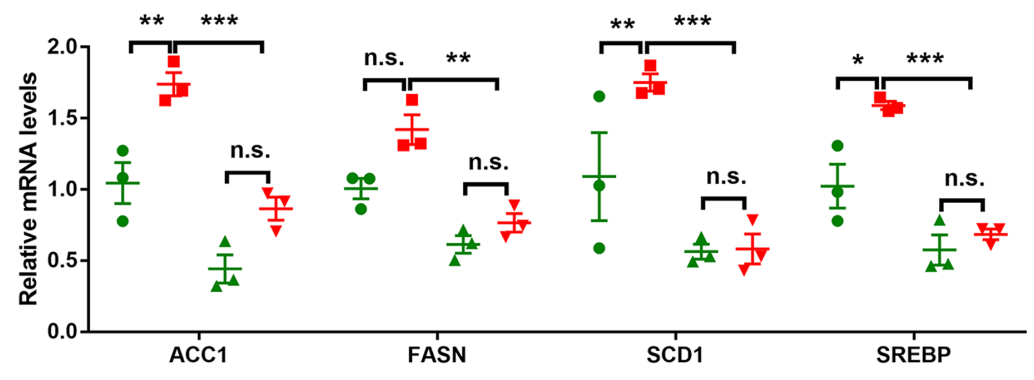

- DMSO+Ad-GFP

- DMSO+Ad-Asprosin

\ Oltipraz+Ad-GFP

$\checkmark$ Oltipraz+Ad-Asprosin

Figure 5

Asprosin promotes adipogenesis in primary adipocytes in vitro through Nrf2 pathway. (A) Relative mRNA expression of lipogenesis-related genes in mice scWAT ( $n=4-8)$. (B and C) Lipogenesis was rescued by oltipraz treatment in Ad-Asprosin cells $(n=3)$. (B) Western blot analysis of ACC1 and FASN in the scWAT of experimental mice $(n=5)$. (C) Representative images of ORO staining in adipocytes treated with Ad-GFP or Ad-Asprosin, with or without oltipraz treatment, was performed on day 8 after induction of differentiation. Scale bar is $50 \mu \mathrm{m}$. Quantification of relative ORO-stained area is shown in the graphs at right. (D) Lipogenesis-related genes were analyzed by RT-qPCR. The temperature condition was RT. $* P<0.05, * \star P<0.01$ and $* \star \star P<0.001$ compared to the control group. n.s. means no statistical significance. A full color version of this figure is available at https://doi.org/10.1530/JOE-20-0503.

Accordingly, the regulation of overexpression of asprosin on lipid synthesis in adipocytes with or without oltipraz treatment was also determined. As shown in Fig. 5D, the adipogenic gene expressions were upregulated in adipocytes with asprosin overexpression, but the trend was reversed with Nrf2 activation. Altogether, these findings indicate that asprosin not only inhibits browning and thermogenesis of adipose tissue, but also can aggravate lipid deposition in adipocytes.

https://joe.bioscientifica.com https://doi.org/10.1530/JOE-20-0503

2021 The authors Published by Bioscientifica Ltd. Printed in Great Britain

\section{Discussion}

White adipocytes play a key role in maintaining wholebody energy homeostasis by forming white adipose tissue (WAT) (Schneider \& Chan 2013). Browning of WAT has been recognized as an effective strategy for the treatment of obesity or adiposity (Li et al. 2017). Adipose tissue, as an endocrine organ, can secrete a variety of metabolic regulators, among which adipokines such as adiponectin 
and leptin have been proved to be related to the browning of white adipose tissue (Dodd et al. 2015, Hui et al. 2015). Asprosin is such a newly discovered adipokine thatin is mainly secreted by white adipose tissue (Dodd et al. 2015). Studies on asprosin mainly focused on glucose homeostasis (Li et al. 2019), it has been reported that asprosin is related to a range of metabolic diseases such as non-alcoholic fatty liver disease (Ke et al. 2020), insulin resistance (Yuan et al. 2020), but its role in adipose tissue remains unknown.

In this study, we found that the expression level of asprosin was significantly downregulated in subcutaneous white adipose tissue of HFD-fed or coldstimulated mice, which seems to be contradicted from several other results reporting elevated 'plasma' asprosin in obese mice, and humans (Duerrschmid et al. 2017). However, when asprosin was overexpressed in the scWAT, the cold-stimulated mice displayed attenuated metabolic phenotype (decreased energy expenditure, $\mathrm{O} 2$ consumption and $\mathrm{CO} 2$ production rate, concomitant with reduced heat production). These phenomenons indicate that asprosin negatively regulates the browning of white adipose tissue. We suggest that asprosin mRNA levels may be reduced through negative feedback.

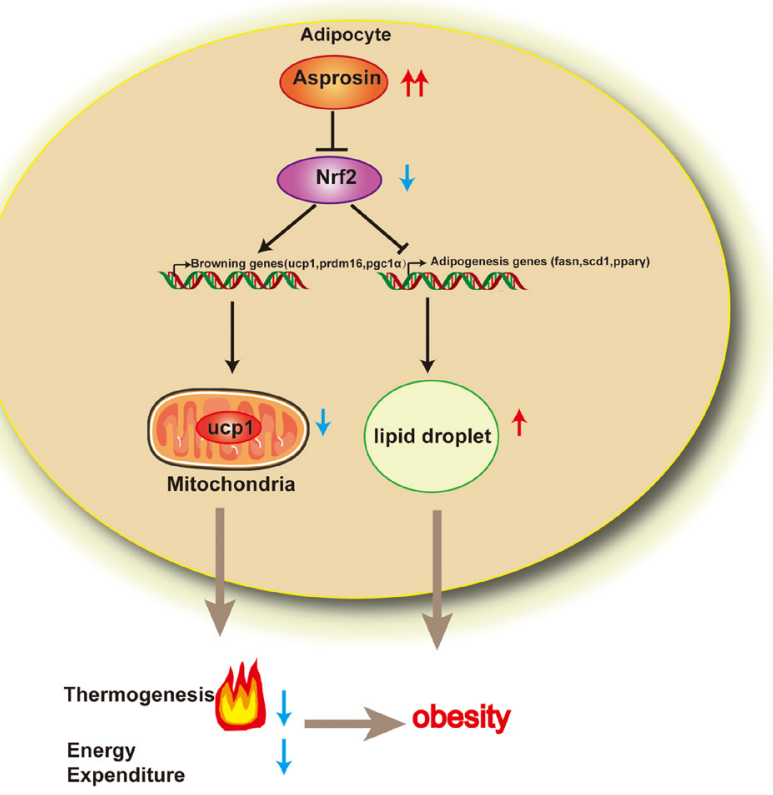

\section{Figure 6}

Asprosin modulates browning and adipogenesis in white adipose tissue through Nrf2-mediated mechanism. Asprosin expression was downregulated during white adipose browning. However, overexpression of asprosin in white adipose tissue inhibited its browning, reduced the body's thermogenesis, increased fat synthesis, and aggravated the lipid deposition in adipocytes by inhibiting the Nrf2 pathway. A full color version of this figure is available at https://doi.org/10.1530/JOE-20-0503.
Consistent with previous studies, we also examined the expression of asprosin and found that the expression of asprosin was increased in adipose tissue of obese $\mathrm{db} / \mathrm{db}$ mice (Supplementary Fig. 1A), which may be the result of negative feedback regulation blocking due to the 'leptin receptor' gene knockout. This finding provided the first evidence that novel adipokine asprosin in adipose tissue may participate in the pathophysiological and physiological processes of obesity.

Mechanistically, overexpression of asprosin suppressed Nrf2 activity in the adipose tissue, which may partially explain the underlying mechanism for asprosin on adipose function. Nuclear factor Erythroid 2-related factor 2 (Nrf2), a member of the NC-BZIP protein family, collaborates with the small muscle fascia fibrosarcoma protein to transcriptional control genes containing antioxidant reaction elements (ARE) (Wang et al. 2020). Nrf2 activation is coordinated by a specific inhibitory factor kelch-like ech-related protein 1 (Keap1), which regulates gene networks and controls a variety of homeostasis processes including adaptive antioxidant reaction and detoxification (Bellezza et al. 2018). Interestingly, a growing body of evidence suggests that Nrf2 may regulate adipose tissue formation and function as a transcription factor, including adipocyte function, lipid metabolism and insulin sensitivity (Wang et al. 2020).

Contrary to our expectations, Nrf2 activity was significantly decreased, while Keap1 expression level showed no significant difference. We speculated that asprosin may directly affect the stability of Nrf2 protein or the transcriptional regulation of its downstream target genes, instead of indirectly affecting Nrf2 expression by adjusting Keap1. Further works need to be done to confirm our guesses.

Nrf2 also exhibits profound effects on adipogenesis. Suppression of Nrf2 activity, genetically or chemically, leads to impaired adipogenesis in preadipocytes ( $\mathrm{Pi}$ et al. 2010, Chen et al. 2013). Consistent with previous studies, mRNA levels of pivotal adipogenic gene expression in scWAT were also upregulated in Ad-Asprosin mice especially upon cold exposure. The increased lipid droplet was further supported by Red Oil O staining. Whereas the Nrf2 agonist oltipraz was administered while overexpressing asprosin, the elevation of lipid deposition in cultured adipocytes was suppressed.

Taken together, mice with asprosin overexpression in scWAT inhibited adipose browning and aggravated lipid deposition in vitro and in vivo, along with the inhibition of Nrf2 activity (Fig. 6). Nrf2 agonist oltipraz could reverse these changes. However, the underlying mechanism needs to be further studied. Another limitation of our study is

This work is licensed under a Creative Commons Attribution 4.0 International License. 
that we did not have an in vivo anti-asprosin antibody treatment, as adverse reactions, including hypersensitivity reactions and renal function impairment, caused by antibody treatment in vivo have been reported in several articles (Bavbek \& Lee 2017, Guo et al. 2018). If a better method can be found in the future, we will further verify the metabolic benefits of asprosin knockdown in vivo.

Our study demonstrated that novel adipokine asprosin is a physiological regulator of adaptive thermogenesis. Overexpression of asprosin in scWAT inhibited browning and energy consumption and increased lipid deposition in adipose tissue, which makes asprosin a potential therapeutic target for obesity and other metabolic disorders.

\section{Supplementary materials}

This is linked to the online version of the paper at https://doi.org/10.1530/ JOE-20-0503.

\section{Declaration of interest}

The authors declare that there is no conflict of interest that could be perceived as prejudicing the impartiality of the research reported.

\section{Funding}

This study was supported by the National Natural Science Foundation of China $(81830014,82000424)$.

\section{Author contribution statement}

The authors have made the following declarations about their contributions: Caijun Rao and Yanli Miao conceived and designed the experiments. Yanli Miao, Haojie Qin, and Yi Zhong performed the experiments. Kai Huang and Caijun Rao analyzed the data and contributed reagents/materials/analysis tools. Yanli Miao and Caijun Rao drafted and revised the manuscript. Yanli Miao and Haojie Qin contributed equally to this work.

\section{References}

Bartelt A \& Heeren J 2014 Adipose tissue browning and metabolic health Nature Reviews: Endocrinology 10 24-36. (https://doi.org/10.1038/ nrendo.2013.204)

Bavbek S \& Lee MJ 2017 Subcutaneous injectable drugs hypersensitivity and desensitization: insulin and monoclonal antibodies. Immunology and Allergy Clinics of North America 37 761-771. (https://doi. org/10.1016/j.iac.2017.06.001)

Bellezza I, Giambanco I, Minelli A \& Donato R 2018 Nrf2-Keap1 signaling in oxidative and reductive stress. Biochimica et Biophysica Acta: Molecular Cell Research 1865 721-733. (https://doi.org/10.1016/j. bbamcr.2018.02.010)

Bhupathiraju SN \& Hu FB 2016 Epidemiology of obesity and diabetes and their cardiovascular complications. Circulation Research 118 1723-1735. (https://doi.org/10.1161/CIRCRESAHA.115.306825)
Braga M, Pervin S, Norris K, Bhasin S \& Singh R 2013 Inhibition of in vitro and in vivo brown fat differentiation program by myostatin. Obesity 21 1180-1188. (https://doi.org/10.1002/oby.20117)

Camilleri M \& Acosta A 2018 Combination therapies for obesity. Metabolic Syndrome and Related Disorders 16 390-394. (https://doi. org/10.1089/met.2018.0075)

Chen Y, Xue P, Hou Y, Zhang H, Zheng H, Zhou T, Qu W, Teng W, Zhang Q, Andersen ME, et al. 2013 Isoniazid suppresses antioxidant response element activities and impairs adipogenesis in mouse and human preadipocytes. Toxicology and Applied Pharmacology 273 435-441. (https://doi.org/10.1016/j.taap.2013.10.005)

Dodd GT, Decherf S, Loh K, Simonds SE, Wiede F, Balland E, Merry TL, Munzberg H, Zhang ZY, Kahn BB, et al. 2015 Leptin and insulin act on POMC neurons to promote the browning of white fat. Cell 160 88-104. (https://doi.org/10.1016/j.cell.2014.12.022)

Duerrschmid C, He Y, Wang C, Li C, Bournat JC, Romere C, Saha PK, Lee ME, Phillips KJ, Jain M, et al. 2017 Asprosin is a centrally acting orexigenic hormone. Nature Medicine 23 1444-1453. (https://doi. org/10.1038/nm.4432)

Fasshauer M \& Bluher M 2015 Adipokines in health and disease. Trends in Pharmacological Sciences 36 461-470. (https://doi.org/10.1016/j. tips.2015.04.014)

Gomez-Banoy N \& Lo JC 2017 Genetic manipulation with viral vectors to assess metabolism and adipose tissue function. Methods in Molecular Biology 1566 109-124. (https://doi.org/10.1007/978-1-4939-6820-6_11)

Greenhill C 2016 Liver: Asprosin - new hormone involved in hepatic glucose release. Nature Reviews: Endocrinology 12 312. (https://doi. org/10.1038/nrendo.2016.66)

Guo Y, Tian X, Wang X \& Xiao Z 2018 Adverse effects of immunoglobulin therapy. Frontiers in Immunology 9 1299. (https://doi.org/10.3389/ fimmu.2018.01299)

Haider N \& Larose L 2020 Activation of the PDGFRalpha-Nrf2 pathway mediates impaired adipocyte differentiation in bone marrow mesenchymal stem cells lacking Nck1. Cell Communication and Signaling 18 26. (https://doi.org/10.1186/s12964-019-0506-4)

Huang L, Pan D, Chen Q, Zhu LJ, Ou J, Wabitsch M \& Wang YX 2017 Transcription factor Hlx controls a systematic switch from white to brown fat through Prdm16-mediated co-activation. Nature Communications 8 68. (https://doi.org/10.1038/s41467-017-00098-2)

Huerta-Delgado AS, Roffe-Vazquez DN, Gonzalez-Gil AM, VillarrealCalderon JR, Tamez-Rivera O, Rodriguez-Gutierrez NA, Castillo EC, Silva-Platas C, Garcia-Rivas G \& Elizondo-Montemayor L 2020 Serum irisin levels, endothelial dysfunction, and inflammation in pediatric patients with type 2 diabetes mellitus and metabolic syndrome. Journal of Diabetes Research 20201949415 . (https://doi. org/10.1155/2020/1949415)

Hui X, Gu P, Zhang J, Nie T, Pan Y, Wu D, Feng T, Zhong C, Wang Y, Lam KS, et al. 2015 Adiponectin enhances cold-induced browning of subcutaneous adipose tissue via promoting M2 macrophage proliferation. Cell Metabolism 22 279-290. (https://doi.org/10.1016/j. cmet.2015.06.004)

Jung TW, Kim HC, Kim HU, Park T, Park J, Kim U, Kim MK \& Jeong JH 2019 Asprosin attenuates insulin signaling pathway through PKCdelta-activated ER stress and inflammation in skeletal muscle. Journal of Cellular Physiology 234 20888-20899. (https://doi. org/10.1002/jcp.28694)

Ke F, Xue G, Jiang X, Li F, Lai X, Zhang M, Shen Y \& Gao L 2020 Combination of asprosin and adiponectin as a novel marker for diagnosing non-alcoholic fatty liver disease. Cytokine $\mathbf{1 3 4} 155184$. (https://doi.org/10.1016/j.cyto.2020.155184)

Lee T, Yun S, Jeong JH \& Jung TW 2019 Asprosin impairs insulin secretion in response to glucose and viability through TLR4/JNK-mediated inflammation. Molecular and Cellular Endocrinology 486 96-104. (https://doi.org/10.1016/j.mce.2019.03.001)

Li G, Xie C, Lu S, Nichols RG, Tian Y, Li L, Patel D, Ma Y, Brocker CN, Yan T, et al. 2017 Intermittent fasting promotes white adipose

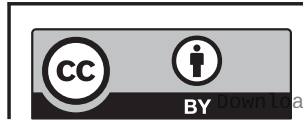

This work is licensed under a Creative Commons Attribution 4.0 International License. 
browning and decreases obesity by shaping the gut microbiota. Cell Metabolism 26 672.e674-685.e674. (https://doi.org/10.1016/j. cmet.2017.08.019)

Li E, Shan H, Chen L, Long A, Zhang Y, Liu Y, Jia L, Wei F, Han J, Li T, et al. 2019 OLFR734 mediates glucose metabolism as a receptor of Asprosin. Cell Metabolism 30 319.e8-328.e8. (https://doi. org/10.1016/j.cmet.2019.05.022)

Longchamp A, Mirabella T, Arduini A, MacArthur MR, Das A, TrevinoVillarreal JH, Hine C, Ben-Sahra I, Knudsen NH, Brace LE, et al. 2018 Amino acid restriction triggers angiogenesis via GCN2/ATF4 regulation of VEGF and H2S production. Cell 173 117.e14-129.e14. (https://doi.org/10.1016/j.cell.2018.03.001)

McQueen AE, Koliwad SK \& Wang JC 2018 Fighting obesity by targeting factors regulating beige adipocytes. Current Opinion in Clinical Nutrition and Metabolic Care 21 437-443. (https://doi.org/10.1097/ MCO.0000000000000509)

Mohammed MS, Sendra S, Lloret J \& Bosch I 2018 Systems and WBANs for controlling obesity. Journal of Healthcare Engineering 2018 1564748. (https://doi.org/10.1155/2018/1564748)

Muthu ML \& Reinhardt DP 2020 Fibrillin-1 and fibrillin-1-derived asprosin in adipose tissue function and metabolic disorders. Journal of Cell Communication and Signaling 14 159-173. (https://doi. org/10.1007/s12079-020-00566-3)

Pi J, Leung L, Xue P, Wang W, Hou Y, Liu D, Yehuda-Shnaidman E, Lee C, Lau J, Kurtz TW, et al. 2010 Deficiency in the nuclear factor E2-related factor- 2 transcription factor results in impaired adipogenesis and protects against diet-induced obesity. Journal of Biological Chemistry 285 9292-9300. (https://doi.org/10.1074/jbc.M109.093955)

Polyzos SA, Kountouras J \& Mantzoros CS 2019 Obesity and nonalcoholic fatty liver disease: from pathophysiology to therapeutics. Metabolism: Clinical and Experimental 92 82-97. (https://doi.org/10.1016/j. metabol.2018.11.014)

Rao C, Huang D, Mao X, Chen R, Huang D \& Huang K 2019 The novel adipokine CTRP5 is a negative regulator of white adipose tissue browning. Biochemical and Biophysical Research Communications $\mathbf{5 1 0}$ 388-394. (https://doi.org/10.1016/j.bbrc.2019.01.111)

Reverte-Salisa L, Sanyal A \& Pfeifer A 2019 Role of cAMP and cGMP signaling in brown fat. Handbook of Experimental Pharmacology 251 161-182. (https://doi.org/10.1007/164_2018_117)
Romere C, Duerrschmid C, Bournat J, Constable P, Jain M, Xia F, Saha PK, Del Solar M, Zhu B, York B, et al. 2016 Asprosin, a fasting-induced glucogenic protein hormone. Cell 165 566-579. (https://doi. org/10.1016/j.cell.2016.02.063)

Salaun H, Thariat J, Vignot M, Merrouche Y \& Vignot S 2017 Obesity and cancer. Bulletin du Cancer 104 30-41. (https://doi.org/10.1016/j. bulcan.2016.11.012)

Schneider KS \& Chan JY 2013 Emerging role of Nrf2 in adipocytes and adipose biology. Advances in Nutrition 4 62-66. (https://doi. org/10.3945/an.112.003103)

Vecchie A, Dallegri F, Carbone F, Bonaventura A, Liberale L, Portincasa P, Fruhbeck G \& Montecucco F 2018 Obesity phenotypes and their paradoxical association with cardiovascular diseases. European Journal of Internal Medicine 48 6-17. (https://doi.org/10.1016/j. ejim.2017.10.020)

Wang R \& Hu W 2020 Asprosin promotes beta-cell apoptosis by inhibiting the autophagy of beta-cell via AMPK-mTOR pathway. Journal of Cellular Physiology 236 215-221. (https://doi.org/10.1002/ jcp.29835)

Wang W \& Seale P 2016 Control of brown and beige fat development. Nature Reviews: Molecular Cell Biology 17 691-702. (https://doi. org/10.1038/nrm.2016.96)

Wang Z, Zuo Z, Li L, Ren S, Gao T, Fu J, Hou Y, Chen Y \& Pi J 2020 Nrf2 in adipocytes. Archives of Pharmacal Research 43 350-360. (https://doi. org/10.1007/s12272-020-01227-0)

Yamamuro T, Kawabata T, Fukuhara A, Saita S, Nakamura S, Takeshita H, Fujiwara M, Enokidani Y, Yoshida G, Tabata K, et al. 2020 Agedependent loss of adipose Rubicon promotes metabolic disorders via excess autophagy. Nature Communications 11 4150. (https://doi. org/10.1038/s41467-020-17985-w)

Yuan M, Li W, Zhu Y, Yu B \& Wu J 2020 Asprosin: a novel player in metabolic diseases. Frontiers in Endocrinology 11 64. (https://doi. org/10.3389/fendo.2020.00064)

Zhao S, Ghosh A, Lo CS, Chenier I, Scholey JW, Filep JG, Ingelfinger JR, Zhang SL \& Chan JSD 2018 Nrf2 deficiency upregulates intrarenal angiotensin-converting enzyme- 2 and angiotensin 1-7 receptor expression and attenuates hypertension and nephropathy in diabetic mice. Endocrinology 159 836-852. (https://doi.org/10.1210/en.201700752)

Received in final form 3 March 2021

Accepted 9 March 2021

Accepted Manuscript published online 11 March 2021 https://joe.bioscientifica.com https://doi.org/10.1530/JOE-20-0503 (c) 2021 The authors Published by Bioscientifica Ltd. Printed in Great Britain

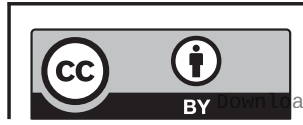

This work is licensed under a Creative Commons Attribution 4.0 International License.

ded from Bioscientifica.com at 04/26/2023 10:33:54AM 